\title{
Feedback Amplifier based on an Embedded HEMT in Thin-film Multilayer MCM-D Technology
}

\author{
Raf Vandersmissen ${ }^{1}$, Dominique Schreurs ${ }^{2}$, Geert Carchon ${ }^{1}$, and Gustaaf Borghs ${ }^{1}$ \\ ${ }^{1}$ IMEC, MCP, Kapeldreef 75, B-3001 Leuven, BELGIUM, +32-16-288056, raf.vandersmissen@imec.be \\ ${ }^{2}$ K.U.Leuven, ESAT/TELEMIC, Kasteelpark Arenberg 10, B-3001 Leuven, BELGIUM
}

\begin{abstract}
In this paper a feedback amplifier circuit integrated in MCM-D (MultiChip Module with Deposited thin films) on glass technology is presented. The active device of the amplifier is a thin-film Ge (germanium) -based HEMT. The HEMT is embedded in the bottom dielectric layer of the MCM-D. The combination of passive MCM-D technology and HEMTs on Ge allows for efficient semimonolithic integration of active devices and realization of MCMs with embedded passive and active components for amplifier circuits.
\end{abstract}

\section{INTRODUCTION}

High packaging density and large scale integration of passive and active components have become key issues in the further development of portable communication systems. Good examples of these trends are the upcoming standards for wideband WLANs (Wireless Local Area Networks) in the 5-6 GHz band. Earlier developments have shown that MCM-D technology can be a viable candidate for the integration and interconnection of high quality microwave and RF applications [1]-[2]. This MCM-D integration of RF (sub-)systems leads to a new implementation paradigm called "System-in-a-Package" (SiP). With this implementation approach, several RF components - each implemented in the most suitable IC technology - can be assembled in a relatively simple and economical way. The passives are as much as possible integrated in the low-cost MCM substrate, hereby reducing the area (and hence the cost) of the active chips.

There are various possibilities to integrate active devices with MCM-D (passive) technology. First, IMEC started with wirebonding GaAs HEMTs on top of a MCM-D carrier substrate. As long wirebonds introduce large parasitic inductances, this method leads to rather poor RF performance. Shifting to flip-chip technology with gold bumps meant shorter interconnect lengths and thus less parasitics and better RF performance. Gold wire studs for thermocompression may be placed at very tight pitches with conventional wirebond equipment [3]. In this paper, a technique for the semi-monolithic integration of an embedded thin-film Ge-based MHEMT (Metamorphic High Electron Mobility Transistor) in MCM-D, is discussed. Compared to GaAs, Ge is cheap and has a low environmental load. The very thin $(<3 \mu \mathrm{m})$ active device is embedded in the MCM-D substrate, together with the passives. In this technique, we use the selective substrate removal method as described in [4]. Due to the conductive nature of $\mathrm{Ge}$, substrate removal is necessary in order to use these devices in microwave circuits.

\section{MCM-D TECHNOLOGY}

Thin-film MCM-Ds are fabricated by a sequential deposition of conductors, typically $\mathrm{Cu}$ or $\mathrm{Al}$, and dielectric layers, typically polyimide or $\mathrm{BCB}$, on a substrate base made of ceramic, silicon or metal. When compared to lower-cost MCM-L (Laminate) and MCM-C (Ceramic) technologies, MCM-D most closely parallels the processing techniques used in the semiconductor industry [3] and offers higher integration densities.

The IMEC MCM-D technology (Fig. 1) consists of alternating thin layers of photosensitive $\mathrm{BCB}$ (Cyclotene $^{\mathrm{TM}}$ from Dow) and low loss $\mathrm{Cu}$ metallizations deposited on a borosilicate glass carrier substrate (AF45, $\left.\varepsilon_{\mathrm{r}}=6.2\right)$. The BCB dielectric has low dielectric losses $\left(\tan \delta \approx 5.10^{-4}\right)$, a low dielectric constant $\left(\varepsilon_{\mathrm{r}}=2.65\right)$ and a low moisture absorption. The material is spin coated in thin films of $5 \mu \mathrm{m}$ thick, then developed and cured. The curing temperature profile of BCB does not exceed $250^{\circ} \mathrm{C}$, which is significantly lower than for most other dielectric materials, e.g., polyimide. Depending on the required metal thickness, the metal layers are sputtered (Al) and patterned using wet etches or electroplated $(\mathrm{Cu})$ on a thin $\mathrm{Ti} / \mathrm{Cu}$ seed layer. The $3 \mu \mathrm{m}$ thick $\mathrm{Cu}$ metal layer is sandwiched between two $30 \mathrm{~nm}$ thick Ti layers rendering good adhesion. The different metal layers are connected through via holes in the $\mathrm{BCB}$ dielectric. Due to the photosensitive property of $\mathrm{BCB}$, the vias are immediately formed after the developing step. The via diameter is typically $30 \mu \mathrm{m}$.

\section{INTEGRATED PASSIVES}

MCM-D is a miniaturization and integration technology capable of high-frequency and high-quality performance.

The resistors indicated in Fig. 1 are realized on the lower level, immediately on the carrier substrate [5]. The resistor material is TaN, with typical resistance values of $10-100 \Omega$ per square and temperature coefficients of less than 20-150 ppm per centigrade.

Integrated capacitors may be realized in different ways [4]. The most common type is the classical MIM (MetalInsulator-Metal) capacitor. The insulating dielectric may be $\mathrm{BCB}$ (capacitance/area ratio $\approx 5.5 \mathrm{pF} / \mathrm{mm}^{2}$ ) or anodized tantalum (capacitance/area ratio $\approx 720 \mathrm{pF} / \mathrm{mm}^{2}$ ) for large values. For the smallest values $(<100 \mathrm{fF})$, interdigital realizations may be used. 
High quality integrated spiral inductors are hard to realize. Especially on standard silicon, the low resistivity of the silicon causes dielectric losses, hereby limiting the quality of the inductors, integrated in a conventional $\mathrm{Al}$ back-end of line, to about 5 at 1 or $2 \mathrm{GHz}$. When realizing the same spiral inductors in MCM-D on a low loss alumina or glass carrier substrate, the quality factor increases tremendously at a lower overall cost [5]. Quality factors above $100 @ 10 \mathrm{GHz}$ (for inductance values $<1.7 \mathrm{nH}$ ) can be realized. The spiral, integrated in the middle $\mathrm{Cu}$ metal layer, is multiturn circular in a coplanar fashion. The center of the spiral is connected to the outside through an overpass using a higher metal layer.

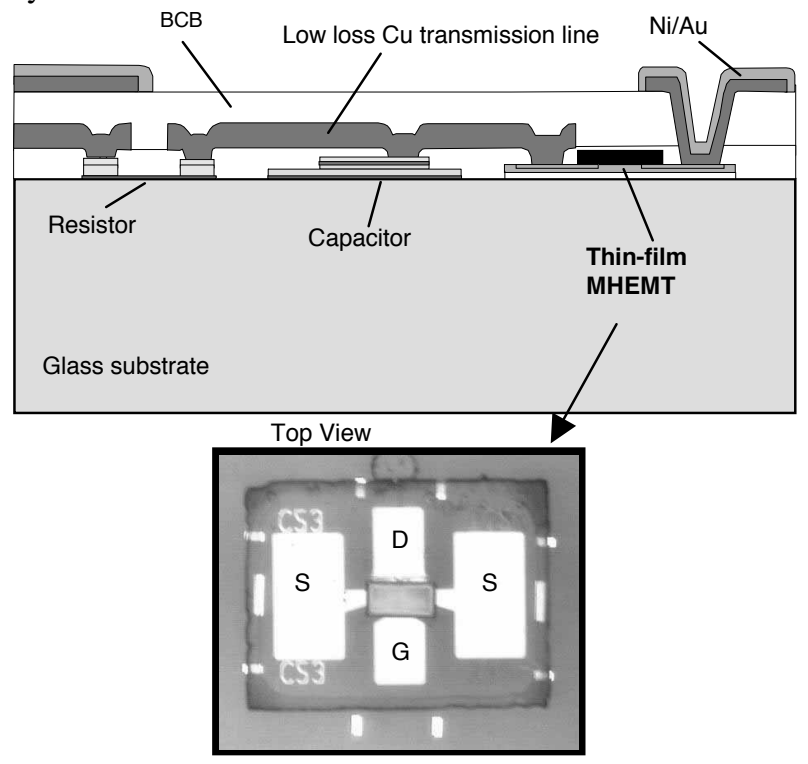

Fig. 1. Schematic cross-section of MCM-D structure with integrated passive and active components; A top view optical micrograph of a thin-film MHEMT after substrate removal.

\section{INTEGRATED ACTIVE DEVICES}

IMEC's MHEMT layers are grown by MBE (Molecular Beam Epitaxy) and devices are fabricated with the same process as used for the production of InP HEMTs [6]. The gate length of the devices is $0.2 \mu \mathrm{m}$ and the gate width is $2 \times 50 \mu \mathrm{m}$. In the case of MHEMTs on cheap Ge substrates, the conductive nature of the substrate makes such devices unsuitable for microwave applications. The substrate, having served its purpose as a platform for growth, is removed and the MHEMT device is transferred to the glass carrier substrate [4]. The thinfilm MHEMTs exhibit excellent DC $\left(\mathrm{g}_{\mathrm{m}}=700 \mathrm{mS} / \mathrm{mm}\right)$ as well as RF $\left(\mathrm{f}_{\mathrm{T}}=80 \mathrm{GHz} ; \mathrm{f}_{\max }=120 \mathrm{GHz}\right)$ performance.

Prior to the removal of the Ge substrate and after deposition of resistor and capacitor layers on the glass carrier substrate, the Ge wafer is diced. A chip containing a single MHEMT is embedded in a spin coated BCB glue layer using a device bonder in thermocompression mode. Chips as small as $400 \times 500 \mu \mathrm{m}^{2}$ can be positioned with 3 $\mu \mathrm{m}$ post-bonding accuracy. After gluing the chip in the
BCB layer, the polymer needs thermal curing for mechanical strength.

Substrate removal and opening of the contact pads of the thin-film MHEMT are done by a highly selective RIE (Reactive Ion Etch) dry etch, a lithography step for protection of the active area of the transistor and wet etching [7]. It is especially important that this etching method does not damage the previously deposited resistor and capacitor layers. A BCB layer of about $5 \mu \mathrm{m}$ thick is then spin coated in order to planarize the structure. In order to circumvent problems in the following processing steps, the total thickness of the devices has to be kept below $5 \mu \mathrm{m}$. This requirement is easily maintained due to the removal of the substrate and buffer layers. Via holes through the BCB dielectric allow to contact the thin-film transistor. A top view optical micrograph of a thin-film MHEMT on glass is shown in Fig. 1. The photograph clearly shows the active area and gold contact pads which are revealed after etching.

Electroplated $\mathrm{Cu}$ transmission lines ensure low loss connections to the different passive and active components. Fig. 1 shows a cross-section of a complete MCM-D build-up with integrated passive and active devices. A BCB layer covers the entire structure and via holes are made. Finally, Ni/Au contact pads are deposited by electroless plating.

\section{Circuit Design AND MEASuREMENT Results}

To demonstrate the abilities of this combination of passive MCM-D technology and thin-film MHEMTs based on $\mathrm{Ge}$, an integrated circuit has been designed and realized. A broadband feedback amplifier has been chosen since these amplifiers can be designed robust enough to allow small changes in the technology. The circuit has been designed using a measurement-based small-signal equivalent model of the thin-film MHEMT and IMEC's integrated passives design library containing parameterized scalable models [8]. The component library is integrated in Agilent's ADS CAE environment. This allows easy and accurate co-design of the active and passive devices. Fig. 2 shows the measured S-parameters for a MHEMT on a combined Smith chart/polar plot. The influence of the conductive $\mathrm{Ge}$ substrate on the $\mathrm{S}$ parameters has completely disappeared after substrate removal.

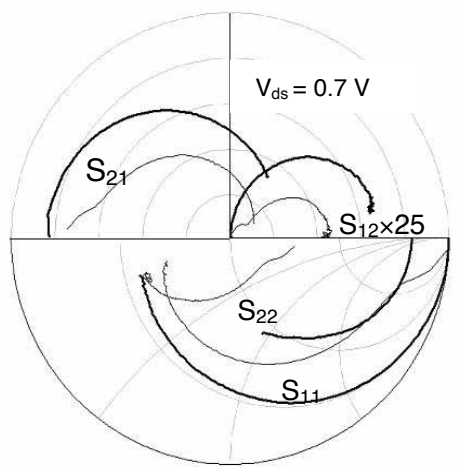

Fig. 2. Measured S-parameters for a MHEMT based on a Ge substrate (combined Smith chart/polar plot; before (thin) and after (thick) substrate removal; $100 \mathrm{MHz}-50 \mathrm{GHz}, 201$ points). 
Fig. 3 shows a photograph of the circuit. The main feedback element is the resistor $\mathrm{R}_{\mathrm{FB}}\left(=\mathrm{R}_{\mathrm{FB} 1}+\mathrm{R}_{\mathrm{FB} 2}\right)$, connected between the drain and the gate of the transistor to control the amount of negative feedback. This negative feedback flattens the gain, makes the transistor more stable and moves the in- and output impedances closer to the desired $50 \Omega$. The value of $R_{F B}$ is taken as a compromise between gain, bandwidth and reflection.

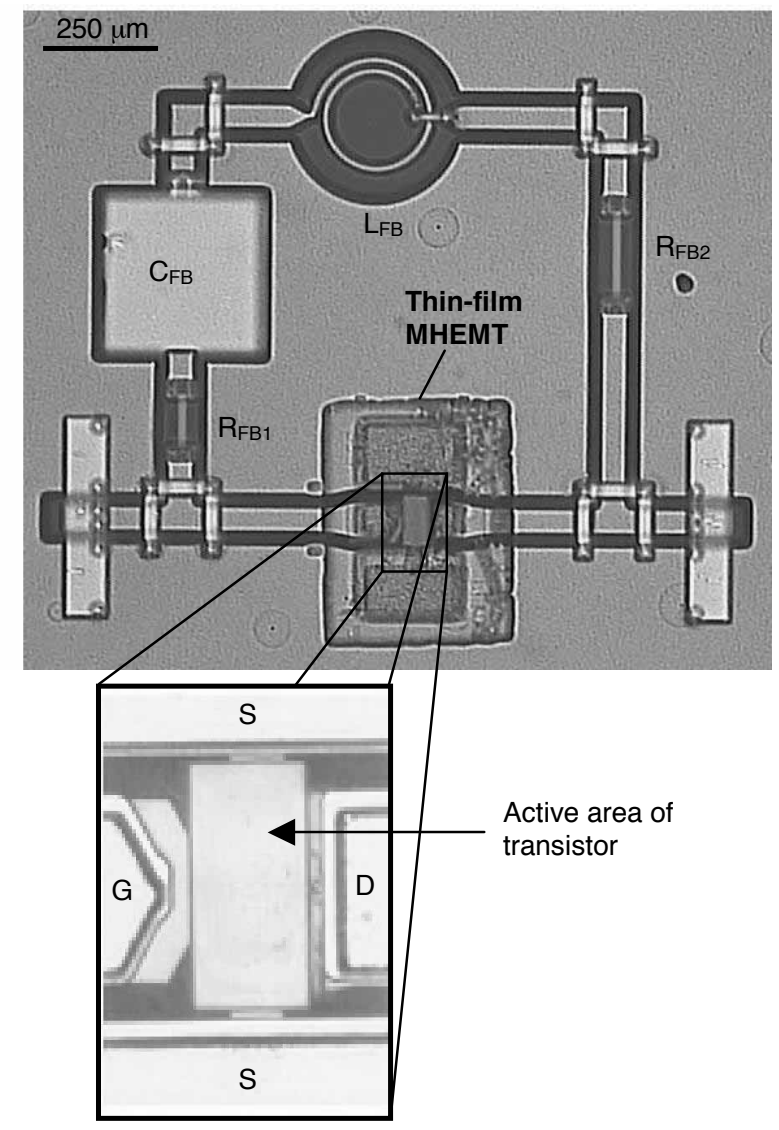

Fig. 3. Optical micrograph of the feedback amplifier on glass (CPW lay-out).

The bandwidth of the amplifier is mainly determined by the gain-bandwidth product of the active device and by the gain controlled by $\mathrm{R}_{\mathrm{FB}}$. Additionally, the feedback loop is made frequency dependent by putting an inductance $L_{F B}$ in series with $R_{F B}$. At the highest frequencies, the inductor reduces the amount of negative feedback thus maintaining a flat gain up to higher frequencies. $\mathrm{L}_{\mathrm{FB}}$ will however also cause an increase of the in- and output reflection at higher frequencies. Finally, a capacitor $\mathrm{C}_{\mathrm{FB}}$ is used to block the DC current between the positive drain and the negative gate bias. Due to the relatively low total transconductance $\left(\mathrm{g}_{\mathrm{m}}=70 \mathrm{mS}\right)$ of the $100 \mu \mathrm{m}$ wide MHEMT used in this design, this amplifier had a relatively low small-signal gain. The low total $\mathrm{g}_{\mathrm{m}}$ also made it difficult to take the value of the feedback resistance $\mathrm{R}_{\mathrm{FB}}$ close to the value for a good inand output matching as for such low $\mathrm{R}_{\mathrm{FB}}$, the gain would drop to almost unity. Instead a relatively high $R_{\mathrm{FB}}$ was taken at the expense of a higher in- and output reflection.

CPW (Coplanar Waveguide) transmission lines, realized on the middle $3 \mu \mathrm{m}$ thick $\mathrm{Cu}$ layer, are used as this has several advantages over the more traditional microstrip approach: through-substrate vias are not required and backside wafer processing can be omitted.

Fig. 4 shows a comparison between the simulated and measured $S_{21}$ of the feedback amplifier. A flat gain of 9 $\mathrm{dB}( \pm 0.5 \mathrm{~dB})$ from 1 to $13 \mathrm{GHz}$ and a very good agreement between measurement and simulation have been achieved. At $1 \mathrm{GHz}$, the input reflection $\left(S_{11}\right)$ is -10 dB.

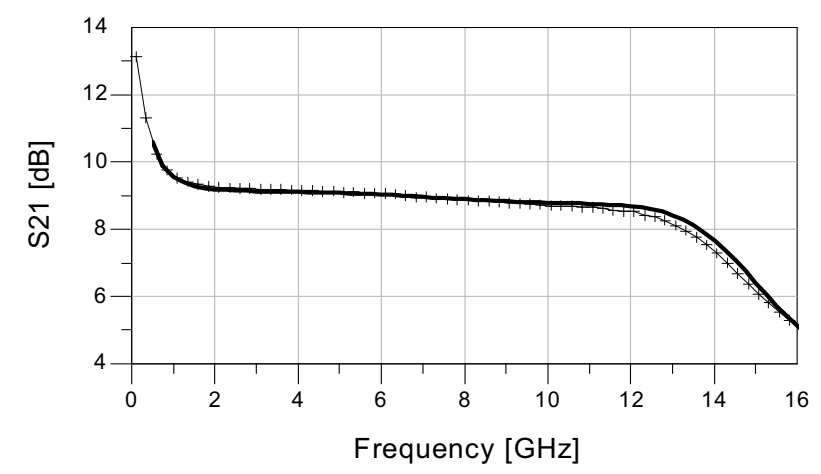

Fig. 4. Simulated and measured (+) $\mathrm{S}_{21}$ of the feedback amplifier.

\section{CONCLUSION}

Thin-film multilayer MCM-D technology using the SiP concept is presented as a viable approach for the integration of high performance passives and embedded active devices. The active devices, i.e., thin-film MHEMTs based on Ge substrates, are embedded in the low-cost MCM-D substrate together with the passives.

A feedback amplifier integrated circuit has been fabricated to demonstrate the abilities of this combination of MCM-D and III-V-on-Ge technology.

\section{ACKNOWLEDGEMENT}

The authors would like to thank P. Mijlemans (Umicore) for supply of Ge wafers and E. Vandenplas, R. Van Hoof and J. Hendrickx for processing. Additionally, financial support from IWT and FWO Flanders was appreciated.

\section{REFERENCES}

[1] R. G. Arnold, C. C. Faulkner and D. J. Pedder, "Silicon MCM-D technology for RF integration", Proceedings of the $6^{\text {th }}$ International Conference and Exhibition on Multichip Modules, pp. 340-344, 1997.

[2] R. C. Frye, P. R. Smith, R. R. Kola, Y. L. Low, M. Y. Lau and K. L. Tai, "Silicon MCM-D with integrated passive components for RF wireless applications", Proceedings of the IEEE MCM Conference, pp. 33-37, 1997.

[3] G. Carchon, K. Vaesen, S. Brebels, W. De Raedt, E. Beyne and B. Nauwelaers, "Multilayer thin film MCM-D for the integration of high-performance wireless front-end systems", Microwave Journal, Vol. 44, pp. 96-110, 2001.

[4] R. Vandersmissen, K. van der Zanden, D. Schreurs and G. Borghs, "Germanium devices: hybrid integration and substrate removal", International Conference on GaAs 
MANufacturing TECHnology (GaAsMANTECH), Technical Digest., pp. 85-88, May 2001.

[5] P. Pieters, W. De Raedt and E. Beyne, "Advances in microwave MCM-D technology", Proceedings of the IMAPS Nordic $36^{\text {th }}$ Annual Conference, Helsinki, 1999.

[6] K. van der Zanden, D. Schreurs, R. Vandersmissen and G. Borghs, "High-performant high-gain amplifiers based on metamorphic GaAs HEMTs", International Conference on GaAs MANufacturing TECHnology (GaAsMANTECH), Technical Digest, pp. 229-232, May 2000.

[7] R. Vandersmissen, D. Schreurs, S. Vandenberghe and G. Borghs, "Optical control of a backside illuminated thin- film metamorphic HEMT", Proceedings of the $32^{\text {nd }}$ European GaAs and related III-V compounds Applications Symposium (GAAS 2002), September 23-25, 2002; Milan, Italy.

[8] G. Carchon, P. Pieters, K. Vaesen, S. Brebels, D. Schreurs, S. Vandenberghe, B. Nauwelaers and E. Beyne, "Designoriented measurement-based scalable models for multilayer MCM-D integrated passives. Implementation in a design library offering automated layout", Proceedings of the International Conference and Exhibition on High Density Interconnection Systems Packaging, pp. 196-201, April 2000. 